Article

\title{
Non-Invasive Biometrics and Machine Learning Modeling to Obtain Sensory and Emotional Responses from Panelists during Entomophagy
}

\author{
Sigfredo Fuentes* $*$, Yin Y. Wong and Claudia Gonzalez Viejo \\ Digital Agriculture, Food and Wine Sciences Group. School of Agriculture and Food, \\ Faculty of Veterinary and Agricultural Sciences, University of Melbourne, Parkville, VIC 3010, Australia; \\ awongyinying@gmail.com (Y.Y.W.); cgonzalez2@unimelb.edu.au (C.G.V.) \\ * Correspondence: sfuentes@unimelb.edu.au; Tel.: +61-03-9035-9670
}

Received: 22 June 2020; Accepted: 7 July 2020; Published: 9 July 2020

\begin{abstract}
Insect-based food products offer a more sustainable and environmentally friendly source of protein compared to plant and animal proteins. Entomophagy is less familiar for Non-Asian cultural backgrounds and is associated with emotions such as disgust and anger, which is the basis of neophobia towards these products. Tradicional sensory evaluation may offer some insights about the liking, visual, aroma, and tasting appreciation, and purchase intention of insect-based food products. However, more robust methods are required to assess these complex interactions with the emotional and subconscious responses related to cultural background. This study focused on the sensory and biometric responses of consumers towards insect-based food snacks and machine learning modeling. Results showed higher liking and emotional responses for those samples containing insects as ingredients (not visible) and with no insects. A lower liking and negative emotional responses were related to samples showing the insects. Artificial neural network models to assess liking based on biometric responses showed high accuracy for different cultures (>92\%). A general model for all cultures with an $89 \%$ accuracy was also achieved.
\end{abstract}

Keywords: entomophagy; neophobia; alternative protein source; emotions; emojis

\section{Introduction}

Increasing public concerns related to the environmental impacts and health-related issues associated with foods from animal sources have influenced the increased research interest in the use and acceptability of alternative protein sources. Insects have been shown as a feasible, sustainable protein source in recent studies [1-5]. The latter is because, for animals, the production of $1 \mathrm{~kg}$ of animal weight will typically require around $2.5 \mathrm{~kg}$ of feed for chicken, $5 \mathrm{~kg}$ for pork, and $10 \mathrm{~kg}$ for beef. On the contrary, for insects, to produce the same weight of crickets (Gryllidae), around $1.7 \mathrm{~kg}$ of feed is required. Furthermore, edible insect parts are around 80\% compared to only $55 \%$ for chicken and $40 \%$ for cattle [6]. Most insects, when compared to plants, have higher protein content-for example, insects have, in general, $35 \%$ to $77 \%$ protein content per edible weight, compared to soybeans with approximately $35 \%$. Insects are considered as a complete protein food due to the presence and amount levels of all, or most of, the essential amino acids required for adequate human health that cannot be found from plant sources, such as cereals and legumes [7,8]. From an environmental point of view, insects can produce between $88 \%$ (cockroaches) and $46 \%$ less $\mathrm{CO}_{2}$ (crickets and beetles) compared to beef cattle per unit of weight gain. Furthermore, the production of other greenhouse gases with 300 and 84 times more potency compared to $\mathrm{CO}_{2}$, such as $\mathrm{N}_{2} \mathrm{O}$ and $\mathrm{CH}_{4}$, respectively, are negligible compared to those produced by beef cattle or pigs [9]. 
Although the consumption of insects dates from ancient times and up to 100 million years ago from anthropological studies, and they are currently a common source of food in more than 100 countries [10,11], most cultures are still reluctant to include them as part of their daily diets [3]. In previous studies, food neophobia and disgust have been reported as the main reasons for consumers being hesitant to try insect-based foods, especially for Westerners [1]. Other studies have reported that curiosity, novelty, and interest in searching for healthier meat alternatives are the main drives for consumers to try insect-based foods [12,13]. It has also been found that consumers are more willing to try these foods when the insects are used as part of their ingredients and are not visible, compared to when they can see the whole insect or parts of them within the presented dish $[1,14]$. Therefore, the initial assessment of the visual attributes of dishes of insect-based foods is very important, as they create the first impression for consumers and determine their eagerness to taste the product or not. Furthermore, the presentation of food, beverages, and even the packaging of food products using imagery presented on digital screens renders statistically similar information when presenting the same product for taste or handling, as it creates the first impression for consumers when judging a product [15-17]. Hence, the visual renderings of insect-based food may help to break negative emotions related to first impressions.

Due to the influence of emotions in decision-making, especially for new food products and even food packaging for consumer acceptability [15,16,18-21], it is important to assess how insect-based food products make consumers feel, besides studying only their acceptability. Therefore, some studies have been conducted to evaluate emotional responses towards foods with insects [22], specifically focused on disgust $[23,24]$. More recently, the use of emojis has been implemented for this purpose, as consumers tend to identify emotions using proxy images related to expressed emotions as non-verbal cues, as these are more closely associated with their feelings [25-27]. Subconscious responses from consumers have also been evaluated for chocolate and beer using computer vision techniques and machine learning to assess their facial expressions $[15,16,20,28,29]$. These studies may produce more relevant information that may be missed using conventional self-reported sensory analysis, especially for insect-based food products.

Therefore, this study aimed to assess consumers' self-reported and subconscious emotional responses towards different insect-based food samples evaluated using emojis, video capturing, and analysis (biometrics) as a quick and reliable approach to understand emotional responses according to cultural backgrounds. To achieve this, a cross-cultural analysis was conducted to compare the responses of Chinese and Australian participants. A sensory session was conducted using the BioSensory application (app; University of Melbourne, Melbourne, Vic, Australia; [30]) to obtain biometrics and check all that apply (CATA), using emojis to assess emotions and a $15 \mathrm{~cm}$ non-structured scale to evaluate the acceptability of five different foods prepared with insects. Furthermore, machine learning (ML) based on artificial neural networks (ANN) models were developed to classify samples into low and high overall liking using the subconscious (biometrics) emotional responses as inputs.

\section{Materials and Methods}

\subsection{Sensory Session Description and Video Analysis}

A total of 88 participants (34 Asians and 54 non-Asians) were recruited from the pool of staff and students at the University of Melbourne (UoM), Australia. The Asian participants were from countries such as China, Malaysia, Vietnam, Philippines, India, and Indonesia, while the non-Asians were from Australia, New Zealand, Mexico, Colombia, Germany, Ukraine, and the United States of America. The ethics considerations were related to the minimal risks associated with personal information and image/video acquisition from participants. Ethics approval was granted by the Faculty of Veterinary and Agricultural Sciences (FVAS) from the UoM with ID: 1545786.2. Furthermore, proper data handling and storage have been followed for security reasons and will be stored for five years. The participants were asked to sign a consent form for them to allow being video-recorded and to specify any allergies 
to assess whether they may take the test. The only information that was provided to participants about the samples along with the consent form previous to the test was "Some samples may contain insects", due to the allergic reactions that these may cause. A power analysis was performed using $S A{ }^{\circledR}$ Power and Sample Size version 14.1 software (SAS Institute Inc., Cary, NC, USA), showing that the number of participants per culture was sufficient to find significant differences among the samples and cultures (Power: $1-\beta>0.99$ ). The session was conducted in individual sensory booths located in the sensory laboratory belonging to the FVAS of the UoM (Melbourne, Australia) using the BioSensory app [30] to display the questionnaire and automatically record videos of the participants while assessing the insect-based food samples. The participants were asked to taste two control and three different insect-based food samples (Table 1), one at a time, and rate their liking for different descriptors as well as to indicate how they felt towards the different samples using a FaceScale (FS; Table 2) and check all that apply (CATA) tests for different emojis that best represented their emotions towards the sample (Table 3). The samples were assigned a 3-digit random number and served at room temperature $\left(20^{\circ} \mathrm{C}\right)$; the avocado was prepared just before serving, and drops of lime juice were added to avoid oxidation and darkening. The participants were provided with water and water crackers as palate cleansers between samples.

Table 1. Image and description of samples used in the sensory session.

\begin{tabular}{cc}
\hline Sample Image & $\begin{array}{c}\text { Sample Description } \\
\text { Tortilla chip with cornflour } \\
\text { (Control) }\end{array}$ \\
\hline \\
Toast with avocado \\
(Control)
\end{tabular}

Table 2. Descriptors and scale used in the questionnaire to acquire self-reported responses. Questionnaires were uploaded in the BioSensory app, including sample numbers, descriptors, scales, and emoticons.

\begin{tabular}{cccc}
\hline Descriptor & Scale & Anchors & Label \\
\hline Appearance & $15 \mathrm{~cm}$ non-structured & $\begin{array}{c}\text { Dislike extremely-Neither like nor } \\
\text { dislike-Like extremely }\end{array}$ & Appearance \\
\hline Appearance & FaceScale (0-100) & & FS App \\
\hline Aroma & $15 \mathrm{~cm}$ non-structured & $\begin{array}{c}\text { Dislike extremely-Neither like nor } \\
\text { dislike-Like extremely }\end{array}$ & Aroma \\
\hline Texture & $15 \mathrm{~cm}$ non-structured & $\begin{array}{c}\text { Dislike extremely-Neither like nor } \\
\text { dislike-Like extremely }\end{array}$ & Texture \\
\hline
\end{tabular}


Table 2. Cont.

\begin{tabular}{cccc}
\hline Descriptor & Scale & Anchors & Label \\
\hline Flavor & $15 \mathrm{~cm}$ non-structured & $\begin{array}{c}\text { Dislike extremely-Neither like nor } \\
\text { dislike-Like extremely }\end{array}$ & Flavor \\
\hline Overall liking & $15 \mathrm{~cm}$ non-structured & $\begin{array}{c}\text { Dislike extremely-Neither like nor } \\
\text { dislike-Like extremely }\end{array}$ & OL \\
\hline Tasting & FaceScale (0-100) & & \\
\hline Purchase intention & $15 \mathrm{~cm}$ non-structured & $\begin{array}{c}\text { Dislike extremely-Neither like nor } \\
\text { dislike-Like extremely }\end{array}$ & PI \\
\hline
\end{tabular}

Table 3. Emojis used for the check all that apply questions for the sensory test using the BioSensory app.

Surprised

Videos were recorded along with the self-reported answers from participants using the BioSensory app and analyzed using a second app developed with the Affectiva software development kit (SDK; Affectiva, Boston, MA, USA). The latter app can analyze videos in batch to obtain the facial expressions from participants based on the micro- and macro-movements of the different features of the face, using the histogram of the oriented gradient for computer vision analysis. Additionally, it uses machine learning algorithms based on support vector machine to translate facial expressions into emotions and emojis; it also assesses head movements (Table 4).

Table 4. Facial expressions and emotion-related parameters obtained from the biometric video analysis (Affectiva app).

\begin{tabular}{cccccc}
\hline Parameter & Label & Parameter & Label & Parameter & Label \\
\hline Joy & Joy & Winking face & Lip Corner Depressor & LCD \\
\hline \multirow{2}{*}{ Disgust } & Disgust & Rage & Lip Press & LPr \\
\hline \multirow{2}{*}{ Sadness } & Sadness & Smirk & Lip Suck & LS \\
\hline
\end{tabular}


Table 4. Cont.

\begin{tabular}{|c|c|c|c|c|c|}
\hline Parameter & Label & Parameter & Label & Parameter & Label \\
\hline Surprise & Surprise & Disappointed & 20 & Mouth Open & MO \\
\hline Anger & Anger & Scared & & Smirk Facial Expression & SmirkFE \\
\hline Fear & Fear & $\begin{array}{l}\text { Stuck out tongue } \\
\text { with winking eye }\end{array}$ & $\bullet$ & Eye Closure & EC \\
\hline Contempt & Contempt & Laughing & $\theta 5$ & Eye Widen & EW \\
\hline Valence & Valence & Kissing & $6 \frac{1}{3}$ & Cheek Raise & CR \\
\hline Smile & Smile & Inner Brow Raise & IBR & Lid Tighten & LT \\
\hline Engagement & Engagement & Brow Rise & BR & Dimpler & Dimpler \\
\hline Attention & Attention & Brow Furrow & $\mathrm{BF}$ & Lip Stretch & LSt \\
\hline Smiley & $\bullet$ & Nose Wrinkle & NW & Jaw Drop & JD \\
\hline Relaxed & $\bullet$ & Upper Lip Rise & ULR & Pitch & \\
\hline Stuck out tongue & $\bullet \bullet$ & Chin Raise & CR & Yaw & \\
\hline Flushed & $\bullet \bullet$ & Lip Pucker & LP & Roll & \\
\hline
\end{tabular}

\subsection{Statistical Analysis and Machine Learning Modeling}

An ANOVA was conducted for the quantitative self-reported and biometric responses with a Fisher's least significant differences (LSD) post hoc test to assess significant differences $(\alpha=0.05)$ between samples nested within cultures (Asians and non-Asians) using XLSTAT ver. 2020.3.1 (Addinsoft, New York, NY, USA). Furthermore, multivariate data analysis was conducted using principal component analysis (PCA) to find the relationships and associations among samples and variables from the quantitative self-reported and biometric responses using Matlab ${ }^{\circledR} \mathrm{R} 2020 \mathrm{a}$ (Mathworks, Inc., Natick, MA, USA). On the other hand, to find relationships between the frequency responses from emojis using the CATA test and quantitative self-reported and biometric responses as well as the associations of samples with each response, a multiple factor analysis (MFA) was conducted using XLSTAT. Furthermore, a correlation matrix was developed using Matlab ${ }^{\circledR}$ R2020a to assess only the significant correlations $(p \leq 0.05)$ between the quantitative self-reported and biometric responses.

The machine learning (ML) models based on artificial neural networks (ANN) pattern recognition were developed using a code written in Matlab ${ }^{\circledR} \mathrm{R} 2020 \mathrm{a}$ to evaluate 17 different training algorithms. Bayesian Regularization was selected as the best algorithm, resulting in models with higher accuracy and no signs of overfitting from performance tests. A total of 45 inputs from the biometrics emotion analysis (Table 4) were used to classify the samples into low and high overall liking. Model 1 was developed using data from Asian participants, while Model 2 was constructed using data from non-Asians. A further ML model (Model 3) was created as a general model, using the results from all the participants regardless of their cultural background. The data were divided randomly using $80 \%$ of the samples (samples $x$ participants) for training and $20 \%$ for testing. The performance assessment was 
based on the mean squared error (MSE), and a neuron trimming test ( $3,7,10$ neurons) was conducted to find the models with the best performance and no overfitting from performance tests. Figure 1 shows the diagram of the ANN models developed using a tan-sigmoid function in the hidden layer and Softmax neurons in the output layer.

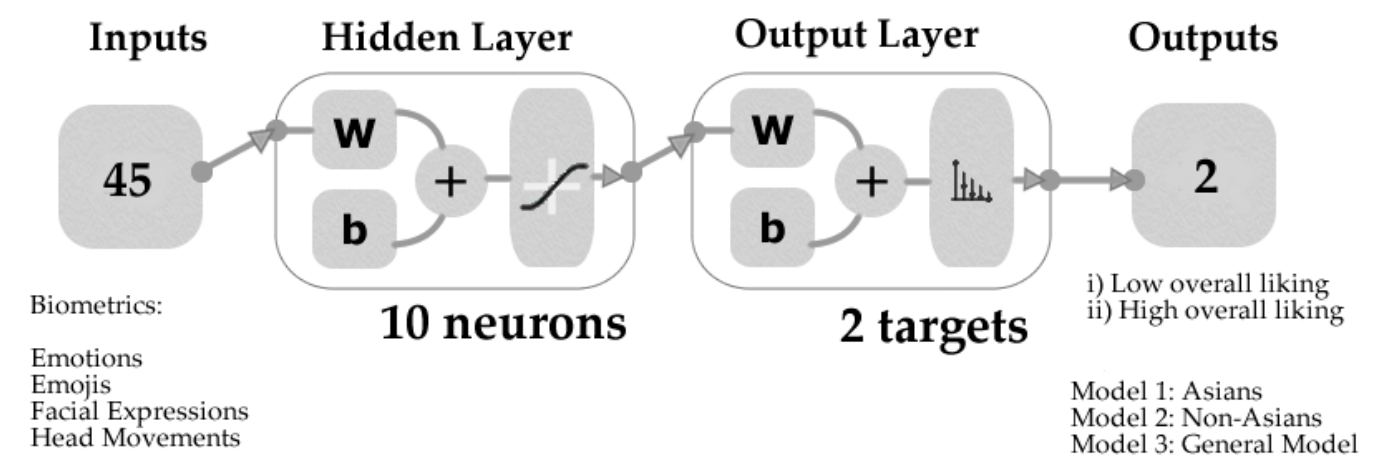

Figure 1. Diagram of the artificial neural network two-layer feed-forward models showing the number of inputs (Table 4), the outputs/targets, and the number of neurons.

\section{Results}

\subsection{Results from the ANOVA of Self-Reported and Biometric Responses}

Results from the ANOVA of the self-reported responses showed that there were significant differences between samples for the eight descriptors considered (Table S1). However, non-significant differences were found between the cultures for appearance, FS App, aroma, texture, and overall liking. For flavor, overall liking, and purchase intention, there were significant differences between cultures for the tortilla chips made with cricket flour and the avocado toast with crickets. Likewise, for FS Taste, there were significant differences between cultures for the avocado toast with crickets. The control samples of tortilla chips and avocado toast, along with the tortilla chips made with cricket flour, were the most liked in terms of appearance and texture. For Asians, besides the control samples, the tortilla chips made with cricket flour were the most liked in terms of texture and overall but were significantly different from the control. Conversely, for non-Asians, the tortilla chips made with cricket flour were amongst the highest in liking of all sensory descriptors as well as in the FaceScale rating, and non-significant differences were found with the control samples. Similarly, for non-Asians, the avocado toast with crickets was amongst the highest in flavor liking, with non-significant differences compared to the control samples.

The results of the ANOVA for the biometrics responses showed there were significant differences between the samples and/or cultures (Table S2). There were significant differences between the cultures

for head roll

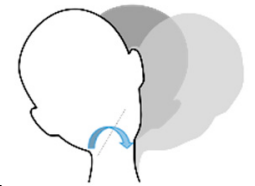

when assessing the avocado toast with crickets and the whole crickets, with more negative results for the Asians, which means that they moved the head to the right side when evaluating these samples. For joy, there were significant differences between cultures, with the avocado toast control sample having higher expression of this emotion in Asians. The Asians also behaved significantly differently to the non-Asians in terms of engagement when assessing the tortilla chips made with cricket flour, with the Asians being more engaged with the sample. The Asians 
the toast with crickets than the non-Asians. Likewise, the Asians expressed significantly more stuck out tongue with winking eye expressions when assessing the tortilla chips made with cricket flour.

\subsection{Multivariate Data Analysis}

\subsubsection{Principal Components Analysis}

Figure 2a shows the PCA from Asians, which explains $74.23 \%$ of the total data variability $(\mathrm{PC} 1=44.18 \%$; PC2: $30.05 \%)$. According to the factor loadings (FL), the principal component one (PC1) was mainly represented by roll $(\mathrm{FL}=0.27)$, flavor $(\mathrm{FL}=0.27)$, and texture $(\mathrm{FL}=0.25)$ on the positive side of the axis, and by winking face $(\mathrm{FL}=-0.26)$, disgust $(\mathrm{FL}=-0.24)$, and sadness $(\mathrm{FL}=-0.24)$ on the negative side. On the other side, PC2 was mainly represented by kissing

$(\mathrm{H}=-0.24)$ on the negative side. On the other side, $\mathrm{PC} 2$ was mainy represented by kissing

$(\mathrm{FL}=0.32)$, rage
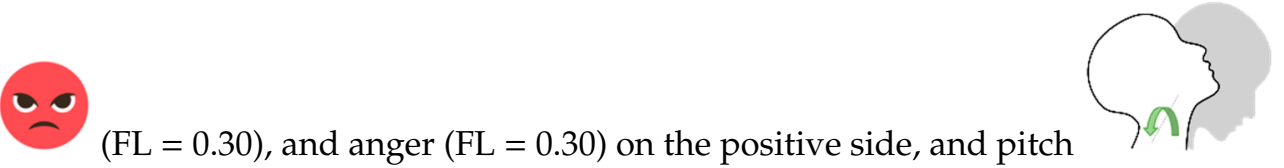

( $F L=-0.19)$ on the negative side of the axis. It can be observed that both control samples (avocado toast and tortilla chip) were associated with a higher liking of flavor, purchase intention, overall liking,

valence, smiley face

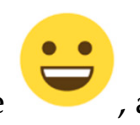
, and roll

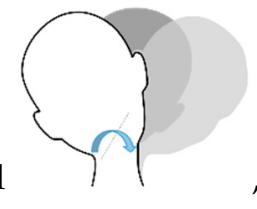

, while the tortilla chip made with cricket flour

was associated with emotions and emojis such as anger, rage

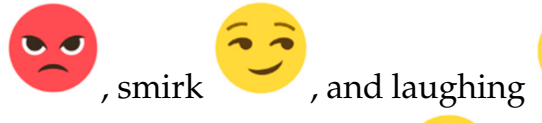

On the other hand, the avocado toast with crickets was associated with winking face

, disgust,

sadness, and flushed face _ - , while the whole crickets were associated with disgust and pitch

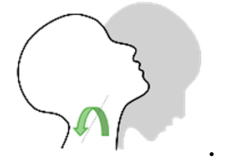

Figure $2 \mathrm{~b}$ shows the PCA for non-Asians, which explained $70.43 \%$ of the total data variability $(\mathrm{PC} 1=50.98 \% ; \mathrm{PC} 2=19.45 \%)$. The PC1 was mainly represented on the positive side of the axis by

joy $(\mathrm{FL}=0.25)$, smiley

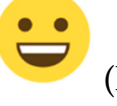

and on the negative side by the self-reported responses $(\mathrm{FL}=0.24)$ appearance, texture, flavor, overall

liking, and purchase intention. The PC2 was mainly represented by pitch

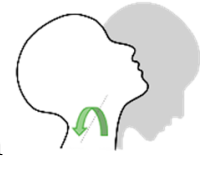

$(\mathrm{FL}=0.38)$ and

stuck out tongue with winking eye

( $F L=0.21)$ on the positive side of the axis, and by surprise

$(\mathrm{FL}=-0.34)$, laughing

$(\mathrm{FL}=-0.34)$, and disappointed

$(\mathrm{FL}=-0.34)$ on the negative side. 
The control sample of the tortilla chip was associated with yaw

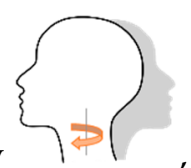
while the control sample of avocado toast and tortilla chip with cricket flour were mainly linked with the liking of aroma and flavor, as well as disgust. On the other hand, the avocado toast with crickets
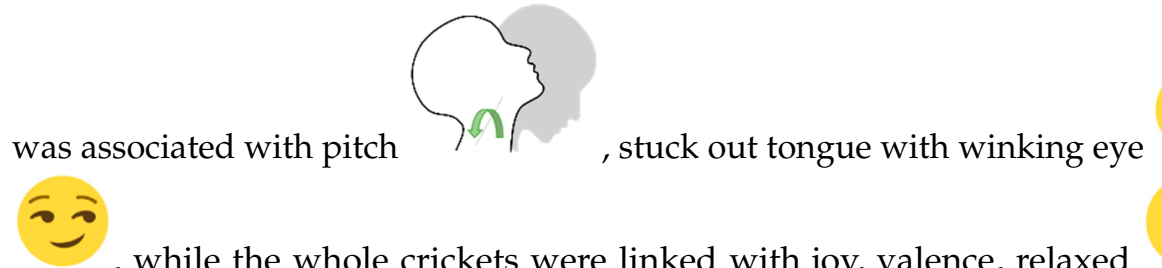

while the whole crickets were linked with joy, valence, relaxed

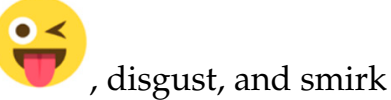
engagement, and attention.

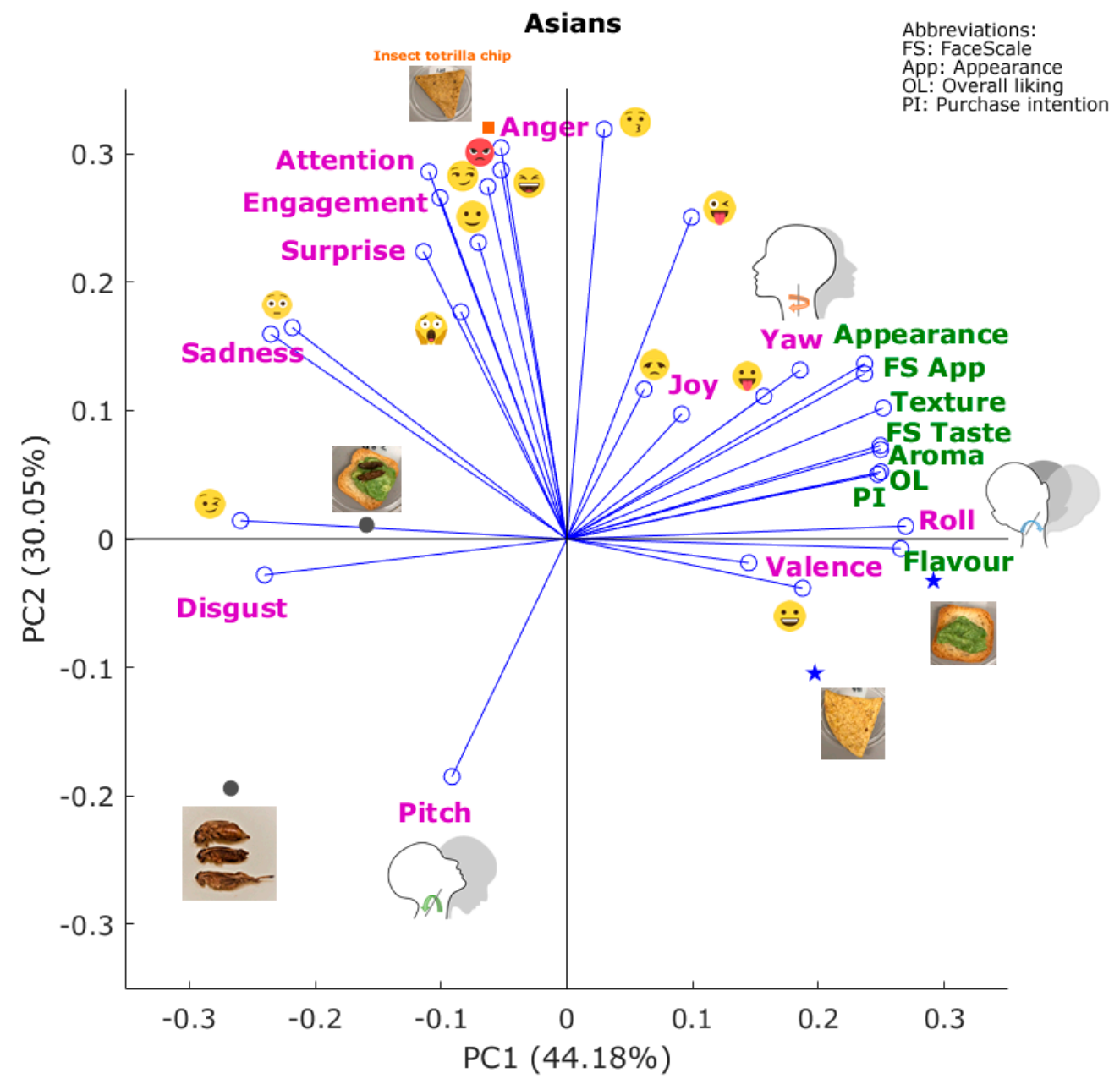

(a)

Figure 2. Cont. 


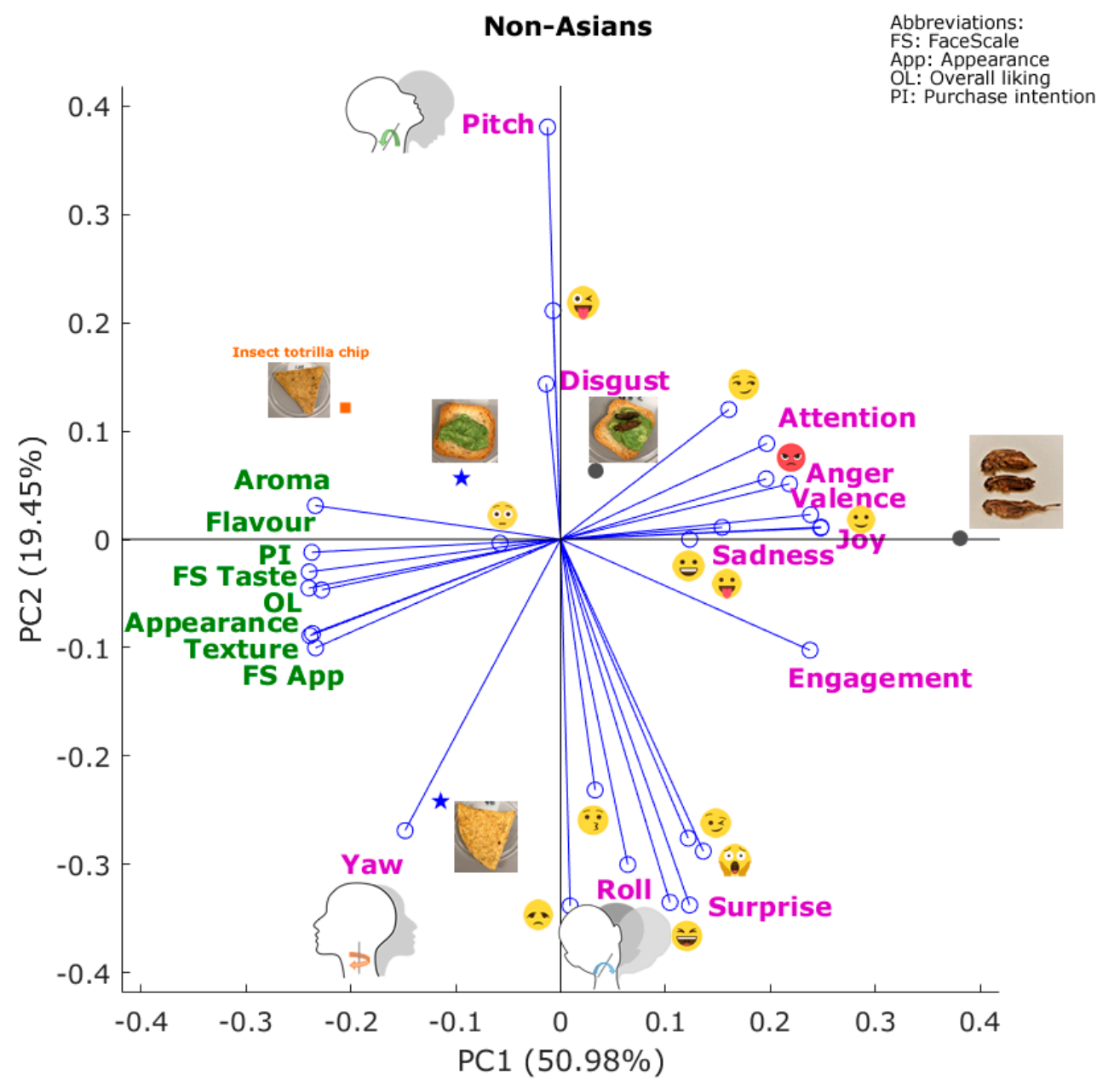

(b)

Figure 2. Principal components analysis for the biometric and quantitative self-reported responses for (a) Asians and (b) non-Asians. Abbreviations: PC1 and PC2: principal components one and two.

\subsubsection{Correlation Analysis}

Figure 3a shows the significant correlations $(p \leq 0.05)$ between the quantitative self-reported and biometric emotional responses for Asians. It can be observed that roll was positively correlated with the liking of appearance (correlation coefficient: $r=0.88)$, aroma $(r=0.92)$, texture $(r=0.93)$, flavor $(r=0.98)$, overall liking $(r=0.92)$, FS Taste $(r=0.91)$, and purchase intention $(r=0.91)$. The liking of aroma

was negatively correlated with winking face

$(r=-0.94)$, and disgust $(r=-0.95)$, while the

liking of flavor had a negative correlation with winking face

$(r=0.93)$, and the latter with FS Taste $(r=-0.88)$. On the other hand, Figure $3 \mathrm{~b}$ shows the significant correlations $(p \leq 0.05)$ found for non-Asians. It can be observed that joy had a negative correlation with the liking of appearance $(r=-0.94)$, FS App $(r=-0.95)$, the liking of texture $(r=-0.97)$, flavor $(r=-0.89)$, overall liking $(r=-0.94)$, FS Taste $(r=-0.89)$, and purchase intention $(r=-0.96)$. Valence had a negative correlation with appearance liking $(r=-0.97)$, FS App $(r=-0.97)$, texture liking $(r=-0.96)$, overall liking $(r=-0.95)$, 
$(r=-0.92)$, FS App $(r=-0.94)$, texture liking $(r=-0.96)$, overall liking $(r=-0.90)$, and purchase intention $(r=-0.93)$. Smiley has a negative correlation with appearance liking $(r=-0.89)$, FS App $(r=-0.88)$, liking of aroma $(r=-0.89)$, texture $(r=-0.91)$, flavor $(r=0.92)$, overall liking $(r=-0.90)$, and purchase intention $(r=-0.90)$.

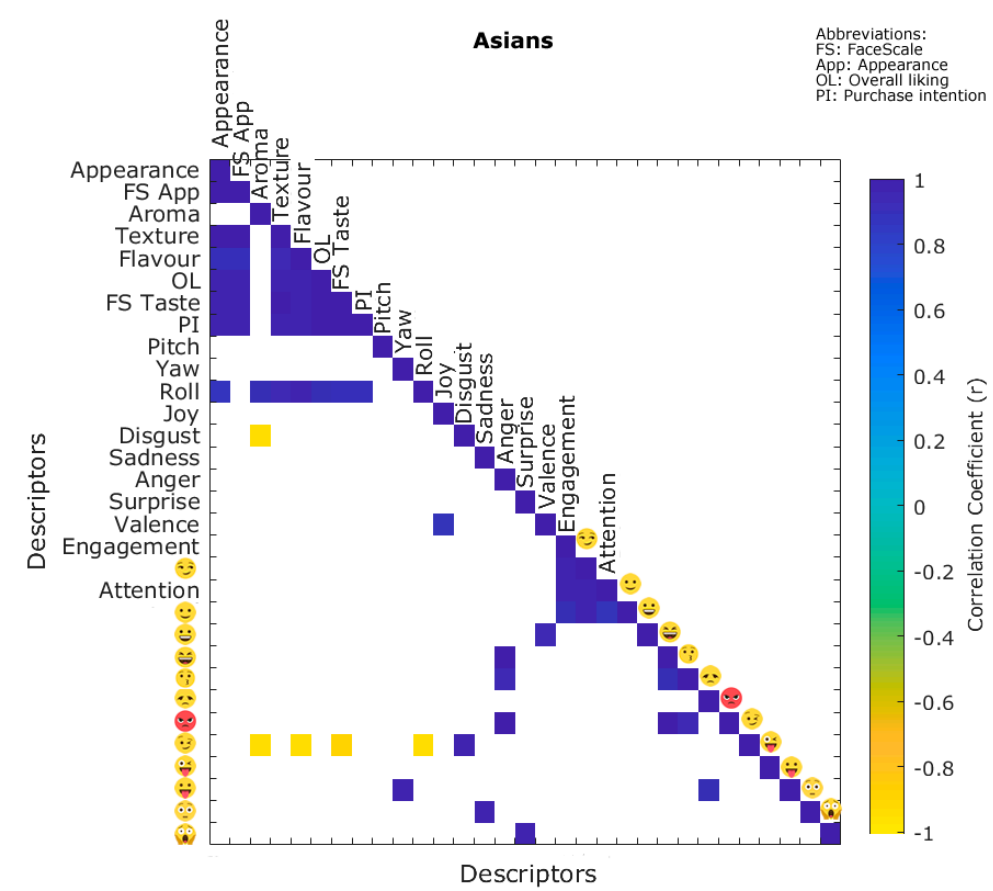

(a)

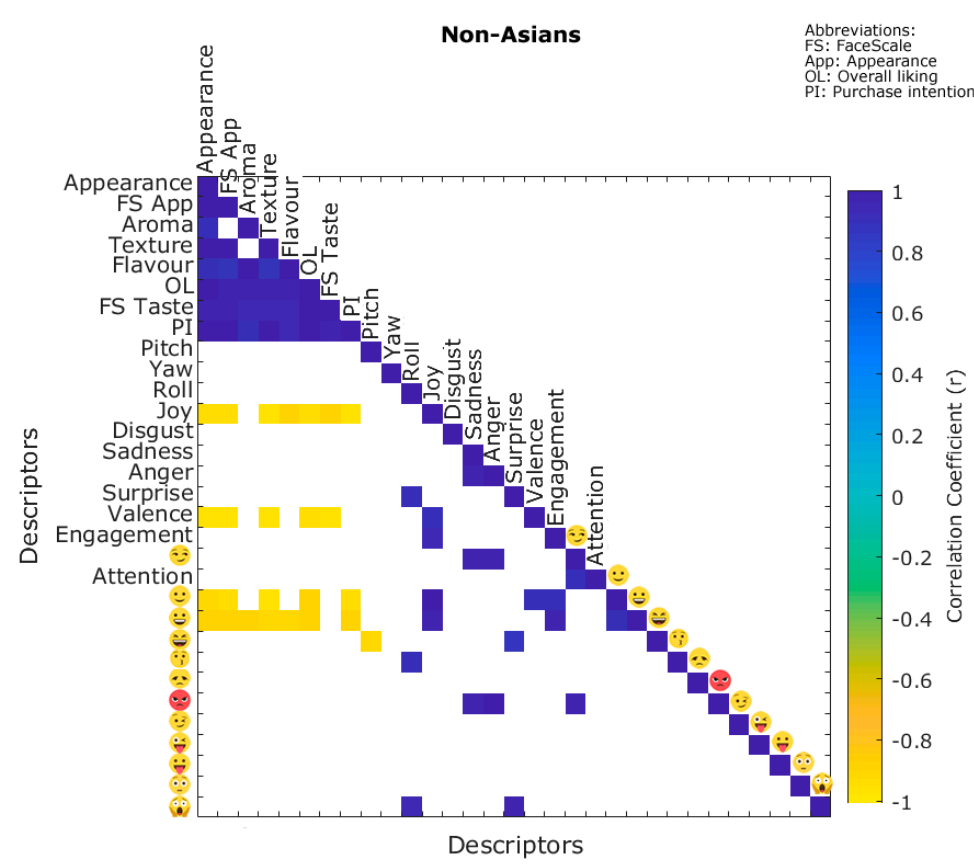

(b)

Figure 3. Matrices showing the significant $(p \leq 0.05)$ correlations between the quantitative self-reported and biometric emotional responses for (a) Asians and (b) non-Asians. Color bar: blue side depicts the positive correlations, while the yellow side represents the negative correlations; likewise, darker blue and yellow denote higher correlations. 


\subsubsection{Multiple Factor Analysis}

Figure 4a shows the MFA of Asian consumers, which explained a total of $76.61 \%$ (Factor 1:

$\mathrm{F} 1=53.69 \%$; Factor $2: \mathrm{F} 2=22.92 \%)$. The F1 was mainly represented by surprised

$(\mathrm{FL}=0.93)$,

and laughing

( $\mathrm{FL}=0.91$ ) on the positive side of the axis, and by savoring

$(\mathrm{FL}=-1.00)$ and appearance liking $(\mathrm{FL}=-0.99)$ on the negative side. On the other hand, F2 was mainly represented on the positive side of the axis by rage $(\mathrm{FL}=0.97)$ and kissing $(\mathrm{FL}=0.93)$, and on the negative side by unamused $(\mathrm{FL}=-0.49)$ and pitch

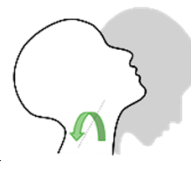

(FL $=-0.42)$. It can be observed that the control samples (tortilla chip and avocado toast) were associated mainly with self-reported responses such as the liking of flavor, aroma, overall liking, and purchase intention, as well as some subconscious responses such as roll
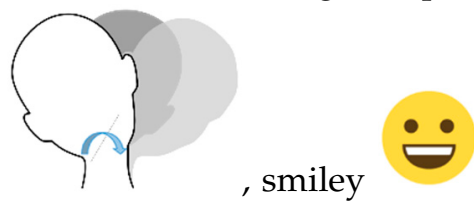
, and valence. The tortilla chip made with cricket flour was associated with emojis measured with biometrics such as kissing laughing from the CATA test. On the other hand, the avocado toast with crickets was associated with emojis from the CATA test, such as disappointed
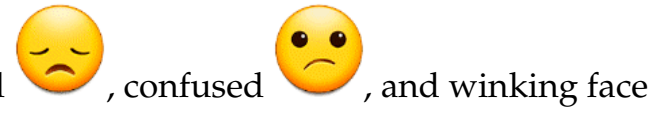

from the

biometrics. The whole crickets were linked to emojis from the CATA test, such as laughing

Figure $4 \mathrm{~b}$ shows the MFA of non-Asian consumers, which explained a total of $76.59 \%$ ( $F 1=58.26 \%$;

$\mathrm{F} 2=18.33 \%$ ). Based on the FL, the F1 was mainly represented by surprised

$(\mathrm{FL}=0.99)$

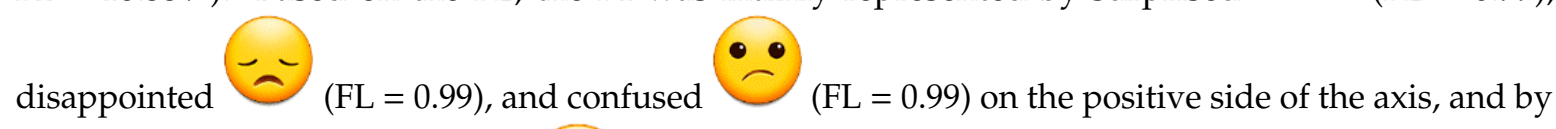

texture liking $(\mathrm{FL}=-1.00)$, happy

$(\mathrm{FL}=-0.99)$, liking of appearance $(\mathrm{FL}=-0.99)$, FS App $(\mathrm{FL}=$ $-0.99)$, and purchase intention ( $\mathrm{FL}=-0.99)$ on the negative side. On the other hand, $\mathrm{F} 2$ was represented

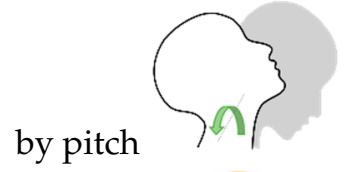


associated with head movements such as yaw

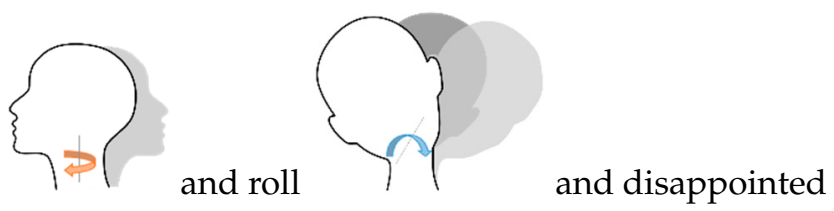

. The control sample of the avocado toast and the tortilla chip made with cricket flour were associated with self-reported responses such as the liking of aroma, flavor, overall liking, and purchase

intention as well as flushed - . The avocado toast with crickets was mainly associated with emojis from the CATA test, such as laughing

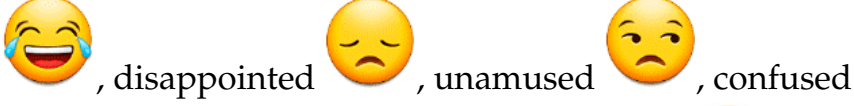
, and neutral and with biometric responses such as valence, stuck out tongue

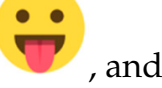

smirk . On the other hand, the whole crickets were linked to the biometric responses such as

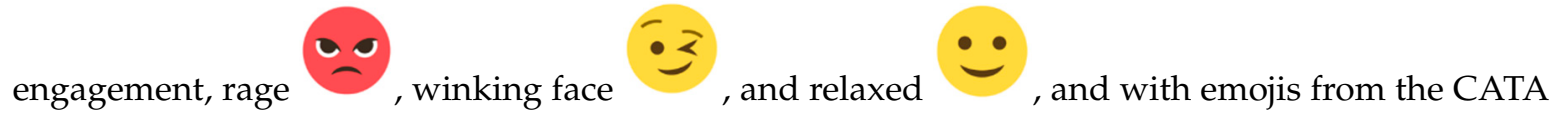
test such as surprised
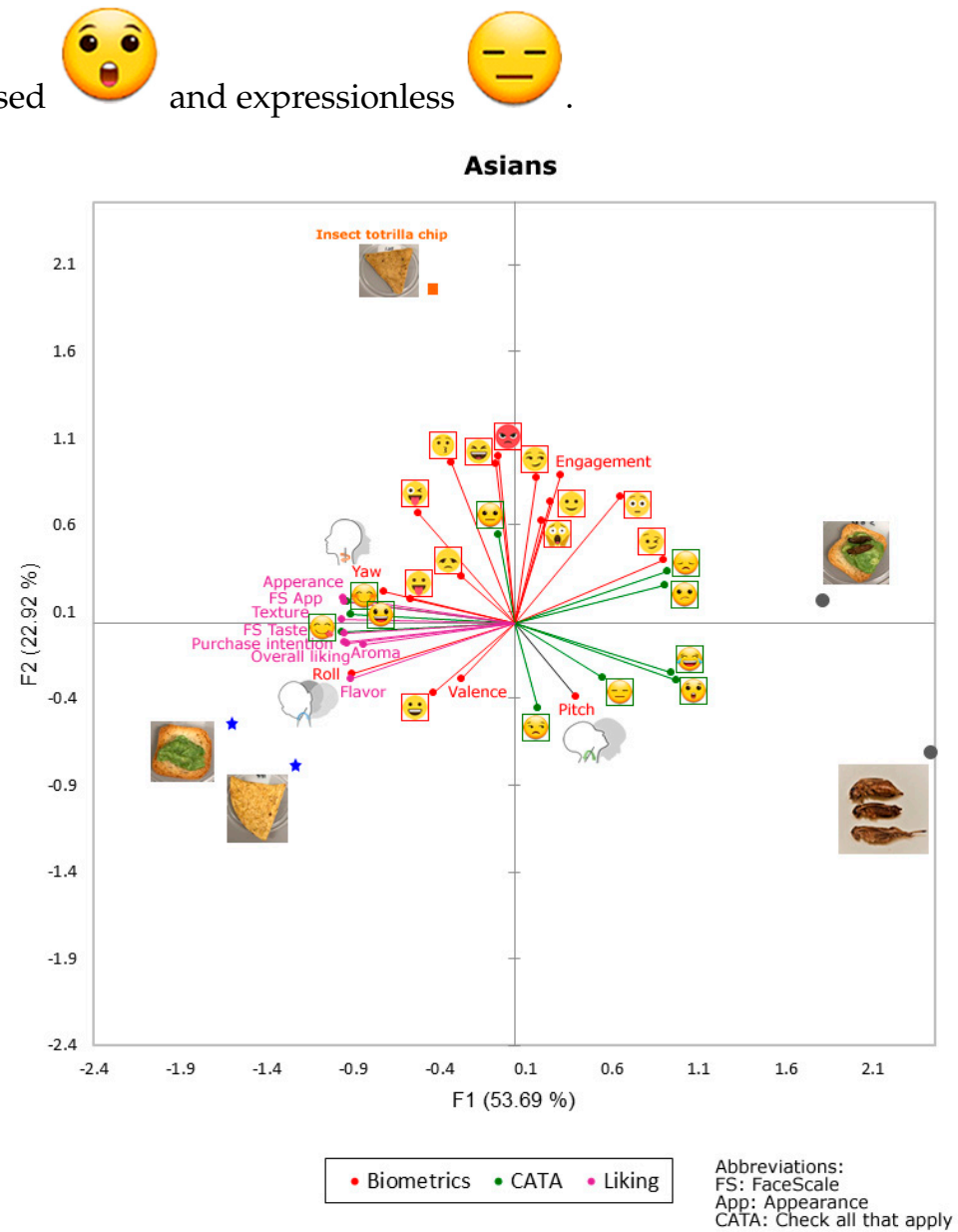

(a)

Figure 4. Cont. 


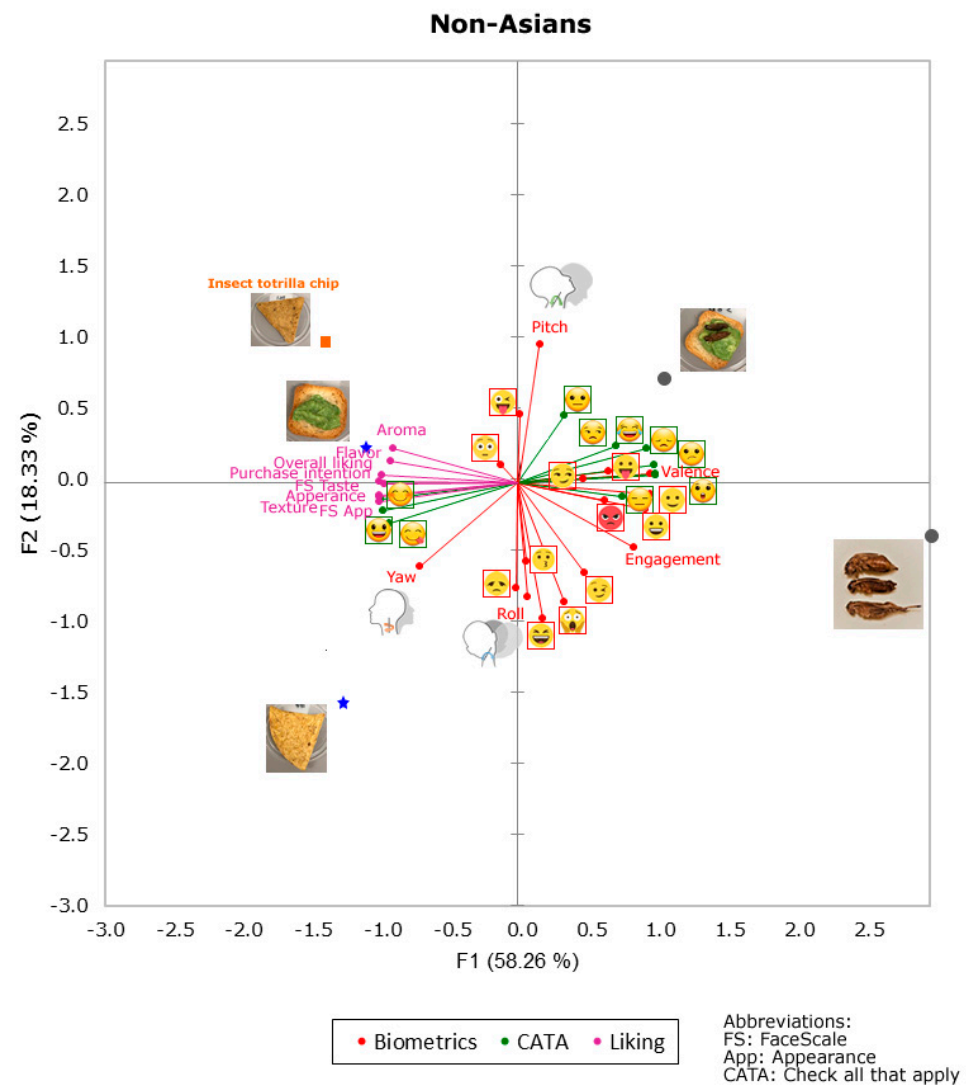

(b)

Figure 4. Multiple factor analysis for the biometric, frequencies (check all that apply: CATA) and quantitative self-reported (Liking) responses for (a) Asians and (b) non-Asians. Abbreviations: F1 and F2: factors one and two.

\subsection{Machine Learning Modeling}

In Table 5, it can be observed that Model 1, developed with the results from the Asian participants, had a $92 \%$ accuracy to classify the samples into high and low liking using only the emotional responses from biometrics as inputs. Model 2 for non-Asians had a higher overall accuracy (94\%) compared to the Asians, and with higher accuracy also in the training stage (non-Asians: 76\%; Asians: $71 \%$ ). On the other hand, Model 3, which was developed as a general model with data from all the participants (Asians and non-Asians), presented an overall accuracy of $89 \%$. The three models had a lower MSE value for the training stage compared to testing, which indicates that there was no overfitting. Figure 5 shows the receiver operating characteristics (ROC) curves, which depict the performance based on the specificity (false positive rate) and sensitivity (true positive rate) of each model and classification group.

Table 5. Statistical data from the three artificial neural network models. Performance shown is based on the mean squared error (MSE).

\begin{tabular}{ccccc}
\hline Stage & Samples $\times$ Participants & Accuracy & Error & $\begin{array}{c}\text { Performance } \\
\text { (MSE) }\end{array}$ \\
\hline Training & 134 & Model 1: Asians & \\
\hline Testing & 34 & $97 \%$ & $3 \%$ & 0.03 \\
\hline Overall & 168 & $71 \%$ & $29 \%$ & 0.24 \\
\hline
\end{tabular}


Table 5. Cont.

\begin{tabular}{ccccc}
\hline Stage & Samples $\times$ Participants & Accuracy & Error & $\begin{array}{c}\text { Performance } \\
\text { (MSE) }\end{array}$ \\
\hline \multicolumn{5}{c}{ Model 2: Non-Asians } \\
\hline Training & 216 & $99 \%$ & $1 \%$ & 0.01 \\
\hline Testing & 54 & $76 \%$ & $24 \%$ & 0.20 \\
\hline Overall & 270 & $94 \%$ & $6 \%$ & - \\
\hline Training & 350 & Model 3: General & \\
\hline Testing & 88 & $97 \%$ & $3 \%$ & 0.03 \\
\hline Overall & 438 & $71 \%$ & $29 \%$ & 0.25 \\
\hline
\end{tabular}

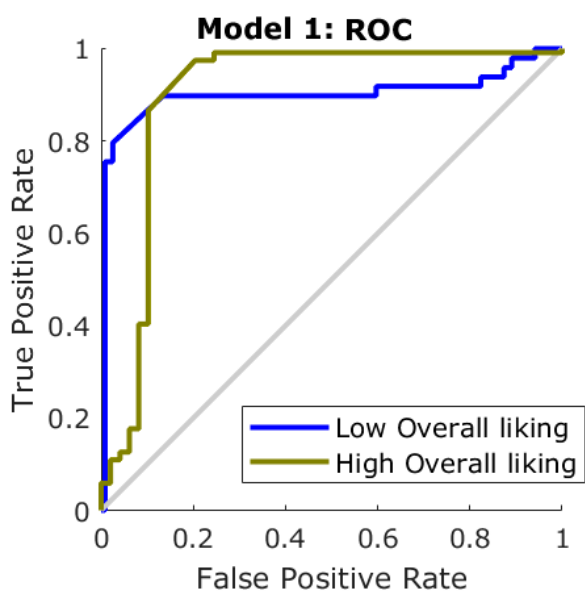

(a)

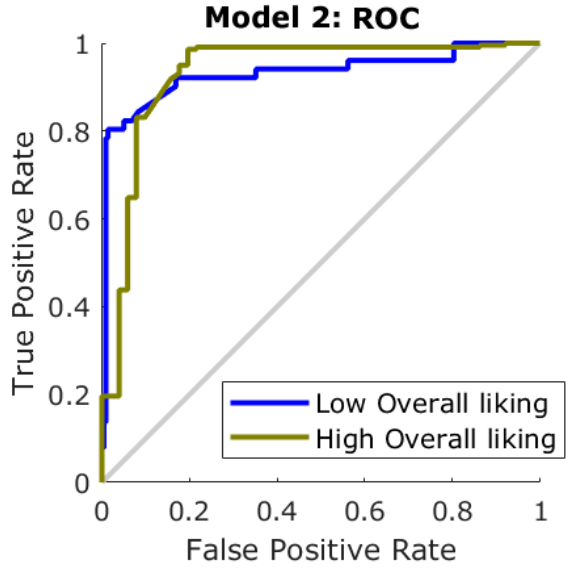

(b)

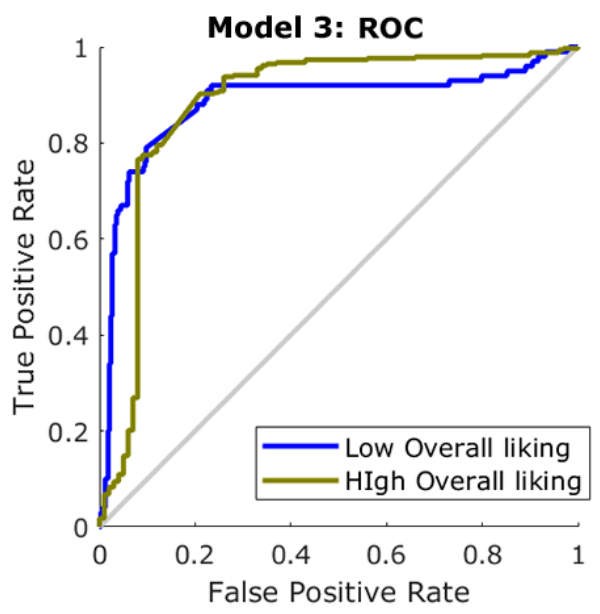

(c)

Figure 5. Receiver operating characteristics (ROC) curves for the three artificial neural network models for (a) Model 1: Asians, (b) Model 2: non-Asians, and (c) Model 3: general (Asians + non-Asians).

\section{Discussion}

In general, for this study, consumer perception had higher scores for self-reported information for the control samples, which did not contain insects, and the lowest scores for full insects, as expected. These scores were similar to the sample with insects as an ingredient, in which no parts of insects were visible, which is in accordance with previous literature [1,14,31]. However, mid-range liking, FS Taste, 
and purchase intention were obtained for visible insects mixed with more familiar products, such as toast and avocado mash (Table S1).

When combining self-reported information with the subconscious and emotional responses through the PCAs (Figure 2), it was found that high and similar variability of data was explained for both the Asian and non-Asian participants ( $>70 \%)$. For the Asians (Figure 2a), more negative emotional responses were associated with insect-based samples compared to the controls, with a clear separation in the vertical plane (PC1). For the non-Asians (Figure 2b), a similar separation was observed for the samples with visible insects, with a difference in the insect tortilla chip. These results may seem contrasting with the self-reported data; however, the subconscious responses are related to the first impression of the samples for visible, aroma, and taste responses from consumers, which are spontaneous and more complex when tasting food and beverage products of any kind [32-34]. Similar relationships were found using the correlation matrix analysis (Figure 3) and the further MFA combining the self-reported data, biometrics, and CATA.

A deeper understanding of consumer acceptability, liking, and intention to purchase new insect-based food products is extremely important, since around $95 \%$ of new food products may fail in the market without proper assessment $[35,36]$. Predictive modeling incorporating cultural backgrounds may offer more information and the possibility of automation for the decision-making process or product variation when developing new insect-based food products. Recent research has been based on automatic estimations of liking based on facial expression dynamics, especially for infants, since self-reported data may not be easily obtained [37,38]. However, research on automatic assessments based on biometrics is rarer [15,28,32].

By using ML modeling considering the separation of cultural background, it was possible to obtain high accuracy $(\geq 92 \%)$ in the prediction of liking based on the biometrics from Asians (Table 5; Figure 5a) and non-Asians (Table 5; Figure 5b) using the Bayesian Regularization ANN algorithm. However, since ML using ANN is considered to be a robust method to detect patterns within data, the cultural distinction could be an internal feature of a general model, as shown in Table 5 and Figure $5 c$. This general model, with an accuracy of $89 \%$, may be used to detect the liking levels of snacks containing insects as part of their ingredients or as whole insects. Further research is required to test and model the biometric responses from a wider variety of insect-based food or beverages.

A quicker analysis for consumer acceptability, liking, and intent to purchase can be achieved as a first approach by presenting images of dishes prepared with insects through the BioSensory app to obtain biometrics from panelists. It has been previously shown for packaging assessments that presenting images of the packaging, and the physical packaging samples resulted in non-significant statistical differences in their appreciation by panelists in a sensory trial [39]. Similarly, for beer tasting panelists looking at videos of beer pouring gave similar levels of liking and general appreciation compared to those that tasted the same beers $[15,40]$. The applicability for the assessment of images of insect-based dishes is supported by the data presented in this study, especially related to the statistically significant correlations between the appearance and overall liking for both cultures (Figure $3 \mathrm{a}, \mathrm{b}$ ) and the higher variability of the data explained by PC1 for both Asians and non-Asians (Figure 2a,b).

The advantages of using both the self-reported and biometric responses rely on the fact that they allow us to obtain the first and subconscious reaction that consumers have towards the product they are assessing as well as the way they may modify their responses after the thinking process. This aids in a deeper assessment of consumers' behavior and acceptability towards food and beverages to understand the target market and develop products according to their needs. The results from Asians and non-Asians were statistically analyzed separately due to the cultural differences that may be related to the expression of emotional responses. This was also the main reason for developing ML models for each culture and a general model considering both cultures. However, despite that Model 3 (general model) had a slightly lower accuracy, it was shown that ANN is able to accurately find patterns among data that may be related to differences in responses from different cultural backgrounds to predict liking regardless. 


\section{Conclusions}

The introduction of insect-based food and beverage products in the market may be a viable alternative for sustainable and nutritional sources of protein. However, the neophobia associated with these products is based on the cultural background and lack of familiarity. More studies are required to break misconceptions and insect-based phobia, which requires the study of complex interactions between the response of consumers to insect-based food products due to visual first impressions, aroma, taste, emotional responses, and cultural background. The implementation of biometrics and machine learning modeling could provide deeper insights regarding these complex interactions and the liking/purchase intention of insect-based food products. Computer vision and machine learning should be further explored and researched as the basis for the development of an artificial intelligence approach to assess new food products and potential success in the market.

Supplementary Materials: The following are available online at http://www.mdpi.com/2304-8158/9/7/903/s1: Table S1: Means and standard error of the self-reported responses for each sample per culture. Different letters denote significant differences based on ANOVA and Fisher's least significant difference post hoc test $(\alpha=0.05)$. Table S2: Means and standard error of the statistically significant biometric responses for each sample per culture. Different letters denote significant differences based on ANOVA and Fisher's least significant difference post hoc test $(\alpha=0.05)$.

Author Contributions: Conceptualization, S.F. and C.G.V.; Data curation, S.F. and C.G.V.; Formal analysis, S.F. and C.G.V.; Investigation, S.F. and C.G.V.; Methodology, Y.Y.W. and C.G.V.; Project administration, S.F. and C.G.V.; Software, S.F. and C.G.V.; Supervision, S.F. and C.G.V.; Validation, S.F. and C.G.V.; Visualization, S.F. and C.G.V.; Writing-original draft, S.F. and C.G.V.; Writing—review and editing, S.F., Y.Y.W. and C.G. All authors have read and agreed to the published version of the manuscript.

Funding: This research received no external funding.

Conflicts of Interest: The authors declare no conflict of interest.

\section{References}

1. House, J. Consumer acceptance of insect-based foods in the Netherlands: Academic and commercial implications. Appetite 2016, 107, 47-58. [CrossRef] [PubMed]

2. La Barbera, F.; Verneau, F.; Amato, M.; Grunert, K. Understanding Westerners' disgust for the eating of insects: The role of food neophobia and implicit associations. Food Qual. Prefer. 2018, 64, 120-125. [CrossRef]

3. Hartmann, C.; Siegrist, M. Insects as food: Perception and acceptance. Findings from current research. Ernahr. Umsch. 2017, 64, 44-50.

4. Lensvelt, E.J.; Steenbekkers, L. Exploring consumer acceptance of entomophagy: A survey and experiment in Australia and the Netherlands. Ecol. Food. Nutr. 2014, 53, 543-561. [CrossRef]

5. Wilkinson, K.; Muhlhausler, B.; Motley, C.; Crump, A.; Bray, H.; Ankeny, R. Australian consumers' awareness and acceptance of insects as food. Insects 2018, 9, 44. [CrossRef]

6. van Huis, A.; Itterbeeck, J.V.; Klunder, H.; Mertens, E.; Halloran, A.; Muir, G.; Vantomme, P. Edible Insects: Future Prospects for Food and Feed Security; FAO: Rome, Italy, 2013.

7. Rumpold, B.A.; Schlüter, O. Insect-based protein sources and their potential for human consumption: Nutritional composition and processing. Anim. Front. 2015, 5, 20-24.

8. Rumpold, B.A.; Schlüter, O.K. Nutritional composition and safety aspects of edible insects. Mol. Nutr. Food Res. 2013, 57, 802-823. [CrossRef]

9. Oonincx, D.G.; van Itterbeeck, J.; Heetkamp, M.J.; van den Brand, H.; van Loon, J.J.; van Huis, A. An exploration on greenhouse gas and ammonia production by insect species suitable for animal or human consumption. PLoS ONE 2010, 5, e14445. [CrossRef]

10. Kouřimská, L.; Adámková, A. Nutritional and sensory quality of edible insects. NFS J. 2016, 4, $22-26$. [CrossRef]

11. Van Huis, A. Did early humans consume insects? J. Insects Food Feed 2017, 3, 161-163. [CrossRef]

12. Verbeke, W. Profiling consumers who are ready to adopt insects as a meat substitute in a Western society. Food Qual. Prefer. 2015, 39, 147-155. [CrossRef]

13. Sogari, G. Entomophagy and Italian consumers: An exploratory analysis. Prog. Nutr. 2015, 17, 311-316. 
14. Gmuer, A.; Guth, J.N.; Hartmann, C.; Siegrist, M. Effects of the degree of processing of insect ingredients in snacks on expected emotional experiences and willingness to eat. Food Qual. Prefer. 2016, 54, 117-127. [CrossRef]

15. Viejo, G.C.; Fuentes, S.; Howell, K.; Torrico, D.; Dunshea, F. Robotics and computer vision techniques combined with non-invasive consumer biometrics to assess quality traits from beer foamability using machine learning: A potential for artificial intelligence applications. Food Control. 2018, 92, 72-79. [CrossRef]

16. Viejo, G.C.; Fuentes, S.; Howell, K.; Torrico, D.; Dunshea, F. Integration of non-invasive biometrics with sensory analysis techniques to assess acceptability of beer by consumers. Physiol. Behav. 2019, 200, 139-147. [CrossRef]

17. Gunaratne, N.M.; Fuentes, S.; Gunaratne, T.M.; Torrico, D.D.; Francis, C.; Ashman, H.; Viejo, C.G.; Dunshea, F.R. Effects of packaging design on sensory liking and willingness to purchase: A study using novel chocolate packaging. Heliyon 2019, 5, e01696. [CrossRef]

18. Megido, R.C.; Gierts, C.; Blecker, C.; Brostaux, Y.; Haubruge, É.; Alabi, T.; Francis, F. Consumer acceptance of insect-based alternative meat products in Western countries. Food Qual. Prefer. 2016, 52, 237-243. [CrossRef]

19. Smith, T.W. The Book of Human Emotions: An Encyclopedia of Feeling from Anger to Wanderlust; Profile Books: London, UK, 2015.

20. Torrico, D.D.; Fuentes, S.; Gonzalez Viejo, C.; Ashman, H.; Gunaratne, N.M.; Gunaratne, T.M.; Dunshea, F.R. Images and chocolate stimuli affect physiological and affective responses of consumers: A cross-cultural study. Food Qual. Prefer. 2018, 65, 60-71. [CrossRef]

21. Gunaratne, N.M.; Fuentes, S.; Gunaratne, T.M.; Torrico, D.D.; Ashman, H.; Francis, C.; Gonzalez Viejo, C.; Dunshea, F.R. Consumer acceptability, eye fixation, and physiological responses: A study of novel and familiar chocolate packaging designs using eye-tracking devices. Foods 2019, 8, 253. [CrossRef]

22. Schouteten, J.J.; De Steur, H.; de Pelsmaeker, S.; Lagast, S.; Juvinal, J.G.; de Bourdeaudhuij, I.; Verbeke, W.; Gellynck, X. Emotional and sensory profiling of insect-, plant-and meat-based burgers under blind, expected and informed conditions. Food Qual. Prefer. 2016, 52, 27-31. [CrossRef]

23. Castro, M.; Chambers, E. Consumer Avoidance of Insect Containing Foods: Primary Emotions, Perceptions and Sensory Characteristics Driving Consumers Considerations. Foods 2019, 8, 351. [CrossRef] [PubMed]

24. Jensen, N.H.; Lieberoth, A. We will eat disgusting foods together-Evidence of the normative basis of Western entomophagy-disgust from an insect tasting. Food Qual. Prefer. 2019, 72, 109-115. [CrossRef]

25. Jaeger, S.R.; Lee, S.M.; Kim, K.-O.; Chheang, S.L.; Jin, D.; Ares, G. Measurement of product emotions using emoji surveys: Case studies with tasted foods and beverages. Food Qual. Prefer. 2017, 62, 46-59. [CrossRef]

26. Kaye, L.K.; Wall, H.J.; Malone, S.A. "Turn that frown upside-down": A contextual account of emoticon usage on different virtual platforms. Computs. Hum. Behav. 2016, 60, 463-467. [CrossRef]

27. Jaeger, S.R.; Vidal, L.; Kam, K.; Ares, G. Can emoji be used as a direct method to measure emotional associations to food names? Preliminary investigations with consumers in USA and China. Food Qual. Prefer. 2017, 56, 38-48. [CrossRef]

28. Gunaratne, T.M.; Fuentes, S.; Gunaratne, N.M.; Torrico, D.D.; Gonzalez Viejo, C.; Dunshea, F.R. Physiological responses to basic tastes for sensory evaluation of chocolate using biometric techniques. Foods 2019, 8, 243. [CrossRef]

29. Gunaratne, M. Implementation of non-invasive biometrics to identify effects of chocolate packaging towards consumer emotional and sensory responses. Ph.D. Thesis, The University of Melbourne, Melbourne, Australia, 2019.

30. Fuentes, S.; Viejo, G.C.; Torrico, D.; Dunshea, F. Development of a biosensory computer application to assess physiological and emotional responses from sensory panelists. Sensors 2018, 18, 2958. [CrossRef]

31. Menozzi, D.; Sogari, G.; Veneziani, M.; Simoni, E.; Mora, C. Eating novel foods: An application of the Theory of Planned Behaviour to predict the consumption of an insect-based product. Food Qual. Prefer. 2017, 59, 27-34. [CrossRef]

32. Dibeklioglu, H.; Gevers, T. Automatic estimation of taste liking through facial expression dynamics. IEEE Trans. Affect. Comput. 2018. [CrossRef]

33. Zhi, R.; Wan, J.; Zhang, D.; Li, W. Correlation between hedonic liking and facial expression measurement using dynamic affective response representation. Food Res. Int. 2018, 108, 237-245. [CrossRef] 
34. Rocha-Parra, D.; García-Burgos, D.; Munsch, S.; Chirife, J.; Zamora, M.C. Application of hedonic dynamics using multiple-sip temporal-liking and facial expression for evaluation of a new beverage. Food Qual. Prefer. 2016, 52, 153-159. [CrossRef]

35. Buss, D. Food Companies Get Smart about Artificial Intelligence. Food Techinol. 2018, 72, $26-41$.

36. Gonzalez Viejo, C.; Fuentes, S. Beer Aroma and Quality Traits Assessment Using Artificial Intelligence. Fermentation 2020, 6, 56. [CrossRef]

37. Hetherington, M.; Madrelle, J.; Nekitsing, C.; Barends, C.; de Graaf, C.; Morgan, S.; Parrott, H.; Weenen, H. Developing a novel tool to assess liking and wanting in infants at the time of complementary feeding-The Feeding Infants: Behaviour and Facial Expression Coding System (FIBFECS). Food Qual. Prefer. 2016, 48, 238-250. [CrossRef]

38. Nekitsing, C.; Madrelle, J.; Barends, C.; de Graaf, C.; Parrott, H.; Morgan, S.; Weenen, H.; Hetherington, M. Application and validation of the Feeding Infants: Behaviour and Facial Expression Coding System (FIBFECS) to assess liking and wanting in infants at the time of complementary feeding. Food Qual. Prefer. 2016, 48, 228-237. [CrossRef]

39. Torrico, D.D.; Fuentes, S.; Viejo, C.G.; Ashman, H.; Gurr, P.A.; Dunshea, F.R. Analysis of thermochromic label elements and colour transitions using sensory acceptability and eye tracking techniques. LWT 2018, 89, 475-481. [CrossRef]

40. Viejo, G.C.; Torrico, D.; Dunshea, F.; Fuentes, S. Development of Artificial Neural Network Models to Assess Beer Acceptability Based on Sensory Properties Using a Robotic Pourer: A Comparative Model Approach to Achieve an Artificial Intelligence System. Beverages 2019, 5, 33. [CrossRef]

(C) 2020 by the authors. Licensee MDPI, Basel, Switzerland. This article is an open access article distributed under the terms and conditions of the Creative Commons Attribution (CC BY) license (http://creativecommons.org/licenses/by/4.0/). 